\title{
Estudo do padrão de melhora da alopecia androgenética em pacientes com uso de microagulhamento associado a minoxidil e/ou plasma rico em plaquetas e mesoterapia
}

\author{
Study of the pattern of improvement in androgenetic alopecia in patients using \\ microneedling associated with minoxidil and/or platelet-rich plasma and mesotherapy \\ Mario Henrique Quim Ferreira ${ }^{\circledR}$, Isabella Bessegatto Rodrigues ${ }^{\circledR}$, , Marcella Bessegatto Rodrigues ${ }^{\circledR 1}$
}

\section{Resumo}

Introdução: a alopécia ligada a fatores hormonais androgênicos é algo que há muito tempo tem sido alvo de estudos e aplicações de conhecimento científico em todo mundo. Este padrão de perda capilar, principalmente ligada ao sexo masculino dispõe de terapias que atualmente se limitam à interrupção da queda de fios com leve progressão de crescimento em alguns casos. Neste tocante, uma nova técnica de microagulhamento, com ou sem terapias adjuvantes, associado a métodos já consagradas como minoxidil tem levado o tratamento desta alteração estética para melhores níveis de resultado. Objetivo: conduzir uma investigação sobre os resultados atuais de pesquisas que envolvam o microagulhamento associado ao uso de Minoxidil, plasma rico em plaquetas elou mesoterapia. Metodologia: foram feitas pesquisas no banco de dados da PubMed e Scielo em abril de 2021 buscando-se os termos "minoxidil AND microneedling". Resultados e Discussões: os resultados obtidos têm convergido para a proposta de que a associação do método de rolagem (microagulhamento) com a aplicação tópica do vasodilatador (minoxidil) apresenta um padrão de crescimento capilar nunca alcançado pela terapia convencional disponível, e que, se acrescentar plasma rico em plaquetas elou soluções através da mesoterapia, os resultados podem ser ainda mais vantajosos. Conclusão: novos estudos são necessários para abranger as vertentes ainda não elucidadas para o tratamento da alopecia, contudo, os resultados até aqui obtidos são promissores para o sucesso da reposição capilar.

Palavras chave: Microagulhamento, Minoxidil, Alopecia

1. Universidade do Oeste Paulista (UNOESTE). Faculdade de Medicina. Presidente Prudente-SP - Brasil

Trabalho realizado: Universidade do Oeste Paulista (UNOESTE). Faculdade de Medicina. Presidente Prudente-SP - Brasil

Endereço para correspondência: Mario Henrique Quim Ferreira. R. José Bongiovani, 700 - Cidade Universitária - 19050-920 - Presidente Prudente-SP-Brasil.E-mail:mhquim@hotmail.com

\begin{abstract}
Introduction: alopecia linked to androgenic hormonal factors is something that has long been the subject of studies and knowledge applications worldwide. This pattern of hair loss, mainly related to males, has therapies that are currently limited to interruption of hair loss with slight growth progression in some cases. In this regard, a new microneedling technique, with or without adjuvant therapies, associated with well-established methods such as minoxidil has led the treatment of this esthetic alteration to better levels of results. Objective: to conduct an investigation into the current results of research involving microneedling associated with the use of Minoxidil, platelet-rich plasma and/or mesotherapy. Methodology: searches were conducted in the PubMed and Scielo database in April 2021, searching for the terms "minoxidil AND microneedling". Results and Discussions: the results obtained have converged to the proposal that the association of the rolling method (microneedling) with the topical application of a vasodilator (minoxidil) presents a pattern of hair growth never achieved by the available conventional therapy, and that, if added platelet-rich plasma and/or solutions through mesotherapy, the results can be even more advantageous. Conclusion: further studies are needed to cover aspects that have not yet been elucidated for the treatment of alopecia, however, the results obtained so far are promising for the success of hair replacement.
\end{abstract}

Keywords: Microneedling, Minoxidil, Alopecia

\section{Introdução}

A alopecia androgenética (AGA), consiste em uma afecção crônica dermatológica em que ocorre a perda capilar. Apesar de acometer homens e mulheres, é mais prevalente no sexo masculino haja vista sua intima relação com hormônios que dão nome a este padrão de queda ${ }^{(1)}$ e que estão menos presentes no sexo feminino. Atualmente os tratamentos consistem em terapias locais, orais e procedimentos cirúrgicos. 
Os medicamentos mais utilizados são o minoxidil em sua forma tópica e a finasterida oral ${ }^{(2-5)}$.

A AGA é causada por uma combinação de fatores genéticos e hormônios andrógenos. Na mulher também pode ser causada por endocrinopatias associadas a um aumento destes mesmos hormônios ${ }^{(2,6)}$. Atualmente, dentre as etnias, a caucasiana é a que apresenta maior incidência da doença. Cerca de $80 \%$ dos homens caucasianos com cerca de 70 anos de idade apresentam a alteração, já nas mulheres caucasianos de mesma idade a prevalência pode chegar a $42 \%{ }^{(3)}$. Embora possa ser observada alta prevalência em idosos, a doença pode acometer indivíduos na puberdade ${ }^{(3,7)}$.

Os tratamentos atuais visam o controle da alopecia, auxiliam sua estabilização e na recuperação de parte dos fios, mas ainda não há uma-repilação completa após o tratamento ${ }^{(8,1)}$. Terapias medicamentosas como a finasterida ou dudasterida agem impedindo o metabolismo da testosterona em diidrotestosterona $^{(3,9-10)}$. O minoxidil amplamente usado topicamente e mais recentemente via oral tem uma ação importante e reconhecida no tratamento desta afeç̧ão ${ }^{(9-12)}$. Recentemente tem-se esclarecido os diversos mecanismos pelos quais atua a AGA: estiimulando a vasodilatação que aumenta fatores de crescimento locais, ativando prostaglandinas citoprotetoras, diminuindo a inflamação perifolicular, induzindo a via de sinalização Wnt/ $\beta$-catenina, que tem efeitos sobre síntese de DNA e proliferação celular além de uma possível interação antiandrogênica influenciando tanto na fase telógena como na anágena dos fios ${ }^{(3,4)}$.

Outras terapias para o estímulo capilar têm se mostrado presentes na literatura, como vitaminas e aminoácidos, ou ainda fotobiomodulação ${ }^{(4)}$. Dentre estas, uma terapia têm surgido como parte imponente no tratamento da AGA, o microagulhamento. Essa intervenção é realizada através de microperfurações cutâneas em que a técnica é realizada através de microagulhas de aço cirúrgico, variando entre 0,5 e 2,5 $\mathrm{mm}$ de comprimento, que por meio de um rolamento cilíndrico, desliza sobre a pele no estrato córneo ${ }^{(13-14)}$. O comprimento da agulha bem como o número de vezes que a epiderme irá receber as perfurações poderá impactar no sucesso do tratamento. Neste tocante, estudos estão sendo feitos para se estabelecer um protocolo padrão para esta técnica ${ }^{(15-16)}$. O intervalo entre as aplicações de microagulhamento na metodologia dos trabalhos varia entre 1 a 2 semanas.

Acredita-se que o microagulhamento potencializa o efeito de medicamentos utilizados para AGA, agindo na formação de novos vasos sanguíneos (angiogênese), na estimulação de fatores de crescimento derivados de plaquetas e na regeneração celular bem como no aumento de colágeno e elastina ${ }^{(5,17)}$. Estudos mostraram que o microagulhamento facilita a permeação transdérmica induzindo o transporte de substâncias ativas quimicamente através dos níveis cutâneos superficiais. Deste modo, aumenta a concentração do fármaco tornando-o mais efeitvo ${ }^{(5,17-18)}$.

A técnica do microagulhamento em conjunto com outros fármacos para a AGA vem despertando interesse pela possibilidade de gerar melhores resultados do que os tratamentos preconizados atualmente, por apresentar um valor relativamente baixo e ser de fácil realização ${ }^{(16,19-20)}$. Diante dos resultados promissores desse tratamento, e em virtude de sua incipiência, a presente pesquisa visou avaliar o conhecimento disponível sobre a efetividade deste procedimento, buscando os resultados obtidos com a técnica do microagulhamento associada ao uso de minoxidil tópico e aplicação de mesoterapia ou plasma rico em plaquetas (PRP), em comparação com os métodos tradicionais da literatura (minoxidil tópico e finasterida oral) para o tratamento da AGA. Espera-se assim concatenar os conhecimentos já existentes e nortear questões para futuras pesquisas.

\section{Metodologia}

\section{Estratégia Metodológica}

A fim de expor um marcador de qualidade para esta pesquisa, buscou-se realizar os tópicos descritos pela recomendação PRISMA (Preferred Reporting Items for Systematic Reviews and Meta-Analyses) para revisões sistemáticas ${ }^{(21)}$.

Em torno da importância clínica desta abordagem, utilizamos a estratégia PICO (Paciente, Intervenção, Controle, Outcome ${ }^{(22)}$, sendo a ordem da resposta das perguntas deste acrônimo:

- Pacientes: aqueles que sofreram perda capilar por AA;

- Intervenção: tratamento mecânico/químico para AA;

- Controle: comparação de pacientes que receberam aplicação desta técnica e interação com minoxidil tópico 5\%;

- Outcome: avaliação pós terapia do grupo padrão e grupo controle.

\section{Critério de busca}

Foram utilizadas as ferramentas de bancos de dados científicos para a pesquisas sobre as novas técnicas para pacientes com alopécia. As bases bibliográficas selecionadas nesta revisão foram PubMed (US National Library of Medicine Nation alI Institutes of Health) e Scielo (Scientific Eletronic Library Online).

Sabendo-se da necessidade de haver uma linguagem comum para as buscas em bancos de dados, 
utilizamos a ferramenta de indexação e recuperação de assuntos na literatura DeCS (Descritor de Ciência da Saúde) e MeSH (Medical Subject Headings). As palavras de eixo fomentador deste trabalho e que foram aplicadas nas buscas foram "minoxidil" e "microneedling" ou "microagulhamento" para pesquisas no descritor da BVS (Biblioteca Virtual em Saúde). O descritor apresentado após a busca foi apenas "minoxidil" para a pesquisa no MeSH e DeCS. A ausência de descritores envolvendo o agulhamento (em português ou microneedling em inglês) se deve, provavelmente, a esta técnica estar sendo recentemente incorporada nos tratamentos clínicos tegumentares. Todas pesquisas foram realizadas em abril de 2021, sem limitação de data de publicação ou outros filtros do banco de dados do site. Para a junção dos descritores o operador booleano "AND" foi utilizado.

\section{Critério de elegibilidade}

Na análise dos trabalhos, os três autores avaliaram os resultados mostrados pelas buscas. Os dois primeiros avaliaram em conjunto os resultados, enquanto um terceiro autor reavaliou esta filtragem inicial. Para isso, inicialmente foi feita uma avaliação título + resumo. Após estes dois itens serem condizentes com a pesquisa, se observou qual foi a metodologia do artigo.

Quanto ao padrão metodológico do artigo científico, foi dado preferência para trabalhos prospectivo, caso controle, randomizados e/ou duplo cego de pacientes que sofreram perdas capilares por alopecia androgênica e submetidos prioritariamente a análise de microagulhamento por dermaroller com minoxidil, associado ou não a plasma rico em plaquetas (PRP) e/ ou injeções com medicamentos (mesoterapia), e seus resultados no estímulo de folículos em couro cabeludo.

Nesta busca, o banco de dados da Scielo não gerou nenhum desfecho pela pesquisa, tanto os termos em inglês como português, provavelmente devido ao caráter recente desta linha de estudo. Já no PubMed, foram apresentados 30 trabalhos pela busca dos termos "minoxidil AND microneedling", sendo alguns destes trabalhos no padrão de revisões de literatura e de outros formatos que não convergiam com nossas buscas, como microagulhamento por outras técnicas (eletrodinâmico, radiofrequência), feitos em camundongos ou outras divergências, fazendo-se necessária a análise individual dos artigos para homogeneizar o estudo, selecionar e incorporar nesta revisão.

\section{Resultados}

De forma a engrandecer o trabalho, além do eixo fomentador principal (minoxidil isolado ou associado com microlesões por agulhamento), foram escolhidas publicações que implementavam também outros métodos, como adição de plasma rico em plaquetas ou mesosolução tópica (agregado de vitaminas, aminoácidos, minerais e outros componentes).

Após a análise individual dos 30 resultados mostrados pelo PubMed, permaneceram 9 trabalhos que condiziam com uma mesma linha de esclarecimento científico proposto para este tema, conforme algoritmo abaixo (Figura 1).
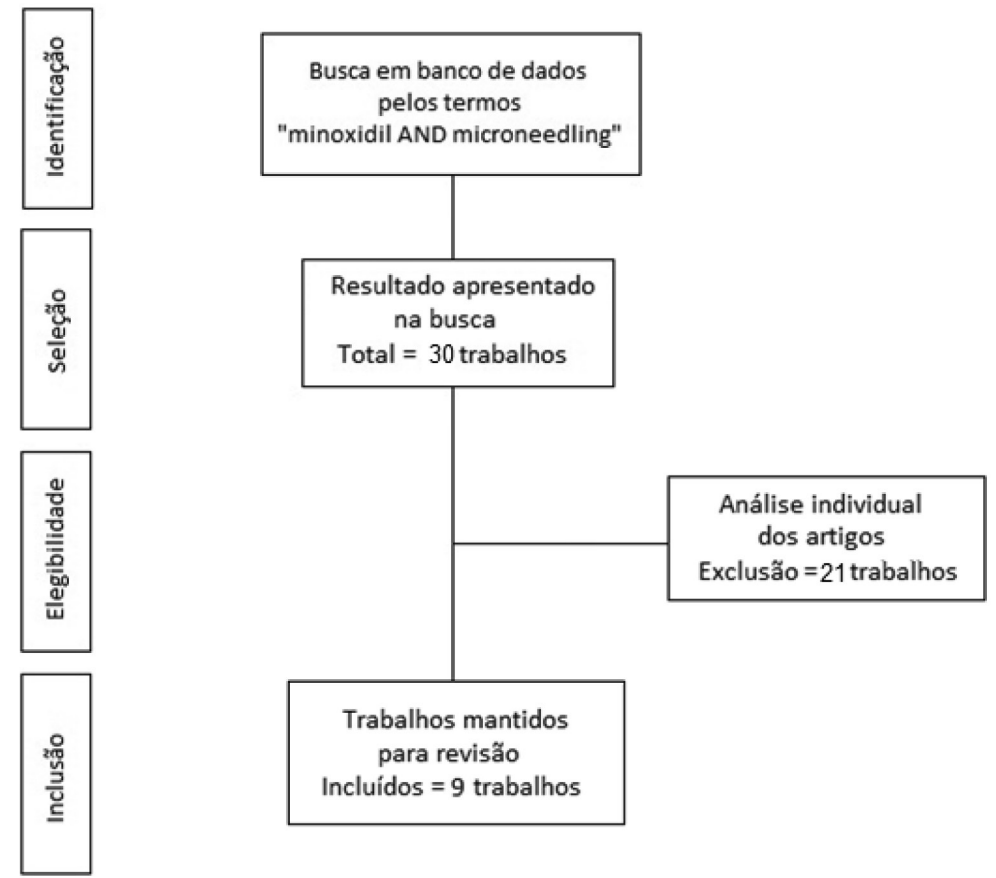

Figura 1 - Algoritmo de busca e seleção artigos em base de dados. 
Nas tabelas a seguir constrói-se uma agregação na análise dos autores nos respectivos trabalhos que condizem a uma linha demonstrativa desta nova terapêutica para AA.
Na segunda tabela compilamos os trabalhos que associam o efeito mecânico do microagulhamento com efeito do plasma rico em plaquetas ou mesosolução tópica (tabela 2). Esta mesolução de forma geral é

\section{Tabela 1}

Trabalhos que avaliam melhora da associação do microagulhamento contra o uso isolado de minoxidil.

\begin{tabular}{|c|c|c|}
\hline Autor & Metodologia & Resultados \\
\hline Kumar et $\mathrm{al}^{(23)}$ & $\begin{array}{l}\text { - Amostra: } 68 \text { pacientes (sexo masculino) } \\
\text { - Tempo de pesquisa: } 12 \text { semanas } \\
\text { - Metodologia: pacientes com grau III e IV de } \\
\text { alopecia androgênica foram divididos em dois } \\
\text { grupos, o primeiro com microagulhamento } \\
\text { semanal + aplicação duas vezes ao dia de } \\
\text { minoxidil 5\%, enquanto o segundo apenas } \\
\text { recebia aplicação da medicação na mesma }\end{array}$ & $\begin{array}{l}\text { O grupo que recebeu terapia combinada teve } \\
\text { um aumento médio na contagem de cabelos de } \\
12,52 \text { / polegada2, enquanto o segundo grupo } \\
\text { teve apenas } 1,89 \text { / polegada2. O segundo teste } \\
\text { aplicado foi o de autoavaliação, sendo que no } \\
\text { primeiro grupo, } 4 \text { pacientes relataram uma } \\
\text { melhora de } 50 \% \text { em comparação com nenhum } \\
\text { do segundo grupo. }\end{array}$ \\
\hline
\end{tabular}
frequência.

Dhurat et $\mathrm{l}^{(24)} \quad$ - Amostra: 4 pacientes (sexo masculino)

-Tempodepesquisa:6meses demicroagulhamento e 18 meses após procedimento.

- Metodologia: Pacientes que já faziam uso de finasterida + minoxidil $5 \%$ porém sem melhora do quadro de crescimento capilar foram submetidos a agulhamentos semanais em couro cabeludo.

\begin{tabular}{ll}
\hline Dhurat et al $^{(25)}$ & - Amostra: 100 pacientes (sexo masculino) \\
& - Tempo de pesquisa: 12 semanas \\
& - Metodologia: estudo comparativo, cego, \\
& durante 12 semanas. 50 pacientes receberam \\
& semanalmente uma sessão de microagulhamento \\
& + minoxidil $5 \% 2 x /$ dia enquanto o segundo grupo \\
& recebeu apenas a solução tópica.
\end{tabular}

Faghihi et $\mathrm{al}^{(26)} \quad$ - Amostra: 60 pacientes (sexos masculino e feminino)

- Tempo de pesquisa: 12 semanas

- Metodologia: este trabalho busca elucidar se há melhora da alopecia com uso de minoxidil associado ao microagulhamento quinzenal e se os melhores resultados são obtidos com 0,6 ou 1,2 mm de profundidade cutânea

Fujita $^{(27)} \quad$ - Amostra: 1 paciente (sexo masculino)

- Tempo de pesquisa: 14 semanas

- Metodologia: foi utilizado neste paciente o uso de minoxidil duas vezes ao dia em couro cabeludo direito, enquanto do lado esquerdo o minoxidil foi associado ao microagulhamento semanal

Sohng et $\mathrm{al}^{(28)}$ - Amostra: 29 pacientes (sexos masculino e feminino)

- Tempo de pesquisa: 6 meses

- Metodologia: foram divididos em 3 grupos para uso dos procedimentos em domicílio: grupo 1 apenas minoxidil; segundo grupo uso de minoxidil e microagulhamento; terceiro grupo apenas microagulhamento
Os pacientes foram acompanhados durante 18 meses após a terapia para avaliar a sustentabilidade da resposta. Na avaliação da escala de 7 pontos, todos os pacientes mostraram um resultado +2 ou +3 . Com isso, o autor concluiu que a resposta foi satisfatória com a adição do agulhamento para pacientes refratários ao tratamento convencional e que esta resposta se manteve ao longo dos meses subsequentes à terapia.

A avaliação por contagem de cabelo mostrou um aumento constante de padrão linear durante as semanas, sendo o coeficiente angular da curva com dermaroller maior do que o do grupo controle $(91,4$ vs 22,2$)$.

40 pacientes do grupo do grupo microneedling tiveram resposta $+2 \mathrm{a}+3$ na escala dos 7 pontos contra nenhum com a mesma melhora do grupo minoxidil.

Foi observado que o microagulhamento com profundidade de $0,6 \mathrm{~mm}$ associado ao minoxidil em termos tanto de contagem de fios como espessura do cabelo, tendendo a ser mais benéfico do que $1,2 \mathrm{~mm}$ de perfuração tegumentar.

No lado onde houve a terapia combinada, o estudo refere que a densidade dos cabelos pelo tricoscópio foi significativamente aumentada em relação ao lado da monoterapia

\footnotetext{
No mês 6 foi observado uma melhora dos resultados no grupo tratamento composto, porém as diferenças observadas não atingiram significância estatística entre os grupos. A autoavaliação do paciente seguiu na mesma vertente, porém, ainda assim o trabalho conclui que o microagulhamento de uso doméstico pode ser uma modalidade terapêutica.
} 

controle (minoxidil).

\begin{tabular}{|c|c|c|}
\hline Autor & Metodologia & Resultado \\
\hline Gajjar et al ${ }^{(29)}$ & $\begin{array}{l}\text { - Amostra: } 49 \text { pacientes (sexo masculino) } \\
\text { - Tempo de pesquisa: } 16 \text { semanas } \\
\text { - Metodologia: durante } 4 \text { meses a população } \\
\text { foi dividida em dois grupos: primeiro grupo } \\
\text { recebeu mesossolução intralesional com microa- } \\
\text { gulhamento em } 8 \text { sessões (grupo A), o segundo } \\
\text { recebeu apenas minoxidil } 5 \% \text { 2x ao dia (grupo B). }\end{array}$ & $\begin{array}{l}\text { Oresultado mostra um aumento da Haste capilar } \\
\text { quando compara o pré e o pós tratamento do } \\
\text { grupo A com grupo B. A observação concluída } \\
\text { pelo autor, no entanto, ressalta que não existe } \\
\text { uma diferença entre mesoterapia e minoxidil }\end{array}$ \\
\hline Jha et $\mathrm{al}^{(30)}$ & $\begin{array}{l}\text { - Amostra: } 93 \text { pacientes (sexo masculino) } \\
\text { - Tempo de pesquisa: } 12 \text { semanas } \\
\text { - Metodologia: os pacientes foram divididos em } \\
3 \text { grupos: A - minoxidil } 5 \% 2 x \text { ao dia; } \mathrm{B} \text { - mino- } \\
\text { xidil 5\% + PRP (em intervalos de } 3 \text { semanas); } \\
\text { C- minoxidil } 5 \% \text { + PRP (intervalos de } 3 \text { semanas) } \\
\text { + microagulhamentos ( } 1,5 \mathrm{~mm} \text { ) }\end{array}$ & $\begin{array}{l}\text { O trabalho mostra um sinergismo entre o plasma } \\
\text { rico em plaquetas e minoxidil. Afirma que sua } \\
\text { associação demonstra resultados superiores ao } \\
\text { uso isolado destes dois compostos. }\end{array}$ \\
\hline Shah et $\mathrm{al}^{(31)}$ & $\begin{array}{l}\text { - Amostra: } 50 \text { pacientes (sexo masculino) } \\
\text { - Tempo de pesquisa: } 6 \text { meses. } \\
\text { - Metodologia: os pacientes foram divididos em } \\
\text { dois grupos, onde o primeiro recebeu apenas } \\
\text { aplicação de minoxidil } 5 \% \text {, enquanto segundo } \\
\text { grupo recebeu minoxidil } 5 \%+\text { microagulha- } \\
\text { mento + PRP. }\end{array}$ & $\begin{array}{l}\text { Pacientes do segundo grupo relataram uma } \\
\text { melhora, tanto em padrão moderado como ex- } \\
\text { celente, quando comparado ao primeiro grupo. }\end{array}$ \\
\hline
\end{tabular}

constituída de vitaminas, aminoácidos, coenzimas, mineiras e/ou ácidos nucleicos ${ }^{(29)}$.

\section{Avaliação dos resultados}

A terapia baseada no uso do minoxidil tópico e finasterida oral atua na redução da perda capilar, com pequeno quantidade aumento no número de fios, mas principalmente agindo no engrossamento da espessura dos mesmos. ${ }^{(24,32-33)}$, sendo o efeito cosmético isolado do minoxidil menos significante. Estes pontos se mostram como uma das causas do abandono da terapia convencional por parte do paciente atualmente ${ }^{(34)}$.

As novas terapias de microagulhamento além da demonstração já experimental de seus bons resultados, tem os seguintes fomentos teóricos para delinear sua funcionalidade ${ }^{(25,35-36)}$.

- Liberação de fatores de crescimento durante a regeneração de feridas cutâneas;

- Ativação de células tronco também na condição de cicatrização das feridas;

- superexpressão de genes relacionados ao crescimento capilar como fator de crescimento vascular, catenina B, Wnt3a e Wnt10b (documentados em estudos em animais).

De uma forma genérica, os estudos têm se embasado em metodologias de microagulhamento com dermaroller de 1,5mm, em frequência de sessões semanais, com 8 rotações por região de couro cabeludo.
Quanto ao minoxidil, sua concentração é de forma geral a 5\%, com duas aplicações diárias.

Os resultados da aplicação do microagulhamento associado ao uso já descrito do minoxidil e/ou PRP e mesosolução parecem gerar efeitos dantes inalcançáveis com tratamento tradicional. Todos os trabalhos que foram avaliados por esta revisão mostraram uma melhora capilar com aumento, em algum nível, no número e/ou espessura de fios com mínimos efeitos colaterais. Em geral os trabalhos utilizaram como avaliação de crescimento a escala dos 7 pontos (Tabela 3), fototricograma, tricoscópio e/ou auto avaliação do paciente.

\section{Tabela 3}

Escala dos 7 pontos de classificação de melhorias.

\begin{tabular}{cc} 
Classificação & Pontuação \\
Piora severa & -3 \\
Piora moderada & -2 \\
Piora branda & -1 \\
Sem mudança & 0 \\
Melhora branda & +1 \\
Melhora moderada & +2 \\
Melhora excelente & +3 \\
\hline
\end{tabular}

Um tocante nesta temática que deve ser enfatizado, é que embora seja positivo os resultados atingidos até o momento, ainda são poucas as publicações e 
Ferreira MHQ, Rodrigues IB, Rodrigues MB. Estudo do padrão de melhora da alopecia androgenética em pacientes com uso de microagulhamento associado a minoxidil e/ou plasma rico em plaquetas e mesoterapia. Relato de caso. Arq Med Hosp Fac Cienc Med Santa Casa São Paulo. 2021; 66:e038.

amostragens encontradas na literatura ${ }^{(37)}$.

Outro ponto a ser destacado são os efeitos colaterais demonstrados nos estudos. Sendo de grande valia as novas ações, obtendo resultados ótimos alcançados por uma terapia relativamente simples, os efeitos colaterais são razoavelmente raros e pequenos como dor local, eritema, cefaleia, sangramento, dermatite seborreica e raramente cicatrizes ${ }^{(31,38-39)}$.

Embora esta revisão tenha como ponto principal a análise do estímulo folicular após as microlesões, também são necessários incorporar os métodos adjuvantes a este que estão sendo estudados, como a aplicação de PRP, que isoladamente já mostrava um resultado benéfico ${ }^{(33-34)}$, e quando aditivado com agulhamento surte efeitos ainda melhores ${ }^{(31)}$.

Além das questões descritas acima, também foi visto que na questão temporal, ao longo das semanas de estudo, a terapia com dermaroller associada ao minoxidil foi estatisticamente superior ao grupo controle de uso isolado da aplicação tópica da solução, sendo sustentado mesmo após meses da pesquisa.

\section{Conclusão}

Técnicas modernas, como o microagulhamento, têm mostrado resultados promissores para o tratamento de AGA, no entanto, a sua eficiência ainda necessita ser testada e respaldada por evidências científicas, tanto na questão amostral como a amplo prazo. A metodologia atual tem se tornado fortalecida e replicada, contudo, não há ainda um mapeamento completo de qual o melhor tamanho de agulha ou frequência de sessões para o tratamento, existindo uma carência de maiores estudos em seres humanos quanto às diferentes técnicas utilizadas e a confirmação da taxa de sucesso que estas podem apresentar.

Baseando-se nos resultados mostrados até aqui, este parece ser um futuro propício na minimização da AGA, um problema de uma sociedade que cada vez mais tem investido em resultados estéticos.

Além disso, convém destacar sobre a necessidade dos pesquisadores também descreverem de forma mais minuciosa os possíveis efeitos adversos da utilização do microagulhamento para alopecia, obtidos através do aumento da quantidade amostral e tempo maior de avaliação do pós procedimento.

Em suma, as microlesões parecem elevar os resultados antes não alcançados pelas técnicas convencionais, sendo uma promessa para os futuros pacientes que irão ser submetidos a tal procedimento, porém, as conclusões das pesquisas com microagulhamento ainda necessitam ser ampliadas para delinear e otimizar o manejo destes procedimentos a fim de diminuir os riscos e atingir resultados cada vez mais próximos do esperado.

\section{Agradecimento}

O nosso agradecimento ao Dr. Baltazar Dias Sanabria que nos auxiliou com várias opiniões da área de atuação capilar durante a produção deste artigo.

\section{Referências}

1. Steiner D, Bartholomei S. Alopecia na mulher. 2013;; 70(10):3439.

2. Rossi A, Anzalone A, Fortuna MC, Caro, G, Garelli V, Pranteda $\mathrm{G}$, et al. Multi-therapies in androgenetic alopecia: review and clinical experiences. Dermatol Ther. 2016; 29(6):424-32.

3. Varothai S, Bergfeld WF. Androgenetic alopecia: an evidencebased treatment update. Am J Clin Dermatol. 2014;15(3):217-30.

4. Gupta AK, Talukder M, Venkataraman M, Bamimore MA. Minoxidil: a comprehensive review.J Dermatol Treat.2021;32:1-11.

5. Fertig RM, Gamret AC, Cervantes J, Tosti A. Microneedling for the treatment of hair loss? J Eur Acad Dermatol Venereol. 2018; 32(4):564-9.

6. Prie BE, Iosif L, Tivig I, Stoian I, Giurcaneanu C. Oxidative stress in androgenetic alopecia. J Med Life. 2016; 9(1):79-83.

7. Blumeyer A, Tosti A, Messenger A, Reygagne, P, Del Marmol V, Spuls $\mathrm{P}$, et al. Evidence-based (S3) guideline for the treatment of androgenetic alopecia in women and in men. J Deutsch Dermatol Ges. [Internet]. 2011 [citado 2021 Jan 22]; 9(suppl. 6):S1-57. Disponível em: https://onlinelibrary.wiley.com/doi/ abs/10.1111/j.1610-0379.2011.07802.x

8. Filbrandt R, Rufaut N, Jones L, Sinclair R. Primary cicatricial alopecia: diagnosis and treatment. Can Med Assoc J. [Internet]. 2013 [citado 2021 Fev 12]; 185(18):1579-85. Disponível em: https://www.cmaj.ca/content/185/18/1579.short.

9. Bartsch G, Rittmaster R, Klocker H. Dihydrotestosterone and the concept of $5 \alpha$-reductase inhibition in human benign prostatic hyperplasia. Eur Urol. 2000; 37(4):367-80.

10. Chiriacò G, Cauci S, Mazzon G, Trombetta C. An observational retrospective evaluation of 79 young men with long-term adverse effects after use of finasteride against androgenetic alopecia. Andrology. 2016; 4(2):245-50.

11. Kaliyadan F, Nambiar A, Vijayaraghavan S. Androgenetic alopecia: an update. Indian J Dermatol Venereol Leprol. [Internet]. 2013 [citado 2021 Jan 23]; 79(5):613-25. Disponível em: https:/ijdvl.com/androgenetic-alopecia-an-update/.

12. Mulinari-Brenner F, Soares IF. Alopecia androgenética masculina: uma atualização. Rev Cienc Med. [Internet]. 2012 [citado 2021 Jan 23]; 18(3):153-61. Disponivel em: http://periodicos.puccampinas.edu.br/seer/index.php/ cienciasmedicas/article/view/642.

13. Singh A, Yadav S. Microneedling: advances and widening horizons. Indian Dermatol Online J. [Internet]. 2016 [citado 2021 Jan 23]; 7(4):244-54. Disponível em: https://www.ncbi.nlm.nih. gov/pmc/articles/PMC4976400/.

14. Lima EVDA, Lima MDA, Takano D. Microagulhamento: estudo experimental e classificação de injúria provocada. Surg Cosmet Dermatol. [Internet]. 2013 [citado 2021 Jan 22]; 5(2):109-14. Disponível em: https://ead.abeb.net.br/pluginfile.php/4721/mod_ glossary/attachment/1/Microagulhamento $\% 20 \% 20$ Estudo $\% 20$ experimental $\% 20 \mathrm{e} \% 20$ classifica $\% \mathrm{C} 3 \% \mathrm{~A} 7 \% \mathrm{C} 3 \% \mathrm{A3o} \% 20 \mathrm{da} \% 20$ inj\%C3\%BAria\%20provocada.pdf.

15. Vermohlem AB, Marques AAF, Belmonte LAO. Microagulhamento associado a fator de crescimento e minoxidil no tratamento da alopecia androgenética: revisão de literatura. Trabalho de Conclusão de Curso (Monografia). Palhoça (SC): UNISUL; 2018. 
Ferreira MHQ, Rodrigues IB, Rodrigues MB. Estudo do padrão de melhora da alopecia androgenética em pacientes com uso de microagulhamento associado a minoxidil e/ou plasma rico em plaquetas e mesoterapia. Relato de caso. Arq Med Hosp Fac Cienc Med Santa Casa São Paulo. 2021 ; 66:e038.

16. Contin LA. Alopecia androgenética masculina tratada com microagulhamento isolado e associado a minoxidil injetável pela técnica de microinfusão de medicamentos pela pele. Surg Cosmet Dermatol. [Internet]. 2016 [citado 2021 Jan 22]; 8(2):158-61. Disponível em: https://pesquisa.bvsalud.org/portal/ resource/pt/biblio-874908?src=similardocs.

17. Strazzulla LC, Avila L, Sicco KL, Shapiro J. An overview of the biology of platelet-rich plasma and microneedling as potential treatments for alopecia areata. J Invest Dermatol. [Internet]. 2017 [citado 2021 Jan 23]; 19(1):21-4. Disponível em: https://www. sciencedirect.com/science/article/abs/pii/S0022202X17327513.

18. Lima AA, Souza TH, Grignoli LCE. Os benefícios do microagulhamento no tratamento das disfunções estéticas. Rev Cient FHO I UNIARARAS. 2015 [citado 2021 Jan 22]; 3(1):92-9. Disponível em: https://cassiacorrea.com.br/ wp-content/uploads/2017/08/6-OS-BENEF\%C3\%8DCIOSDO-MICROAGULHAMENTO-NO-TRATAMENTO-DASDISFUN\%C3\%87\%C3\%95ES-EST\%C3\%89TICAS.pdf

19. Kalil CLPV, Frainer RH, Dexheimer LS, Tonoli RE, Boff AL. Tratamento das cicatrizes de acne com a técnica de microagulhamento e drug delivery. Surg Cosmet Dermatol. [Internet]. 2015 [citado $2021 \mathrm{Fev} 13$ ]; 7(2):144-8. Disponível em: https://www.redalyc.org/pdf/2655/265541072005.pdf.

20. Silva LBP, Santos BA. Uso do laser de baixa intensidade no tratamento da alopecia androgenética: uma revisão bibliográfica. ID On Line: Rev Mult Psicol. [Internet]. 2018 [citado 2021 Fev 13]; 12(40):1065-81. Disponível em: https:// idonline.emnuvens.com.br/id/article/view/1178.

21. Galvão TF, Pansani TDSA, Harrad D. Principais itens para relatar revisões sistemáticas e meta-análises: a recomendação PRISMA. Epidemiol Serv Saúde. [Internet]. 2015 [citado 2021 Fev 14]; 24:335-42. Disponível em: https://www.scielosp.org/ article/ress/2015.v24n2/335-342/pt/.

22. Santos CMDC, Pimenta CADM, Nobre MRC. The PICO strategy for the research question construction and evidence search. Rev Latinoam Enferm. [Internet]. 2007 [citado $2021 \mathrm{Fev}$ 14]; 15(3):508-11. Disponível em: https://www.scielo.br/scielo. php?pid=S0104-11692007000300023\&script=sci_arttext.

23. Kumar MK, Inamadar AC, Palit A. A randomized controlled, single-observer blinded study to determine the efficacy of topical minoxidil plus microneedling versus topical minoxidil alone in the treatment of androgenetic alopecia. J Cutan Aesth Surg. [Internet]. 2018 [citado 2021 Abr 2]; 11(4):211-6. Disponível em: https://www.ncbi.nlm.nih.gov/pmc/articles/PMC6371730/.

24. Dhurat R, Mathapati S. Response to microneedling treatment in men with androgenetic alopecia who failed to respond to conventional therapy. Indian J Dermatol. 2015; 60(3):260-3.

25. Dhurat R, Sukesh MS, Avhad G, Dandale A, Pal A, Pund, P. A randomized evaluator blinded study of effect of microneedling in androgeneticalopecia: a pilotstudy. IntJTrichology.2013;5(1):6-11.

26. Faghihi G, Nabavinejad S, Mokhtari F, Fatemi Naeini F, Iraji F. Microneedling in androgenetic alopecia; comparing two different depths of microneedles. J Cosmet Dermatol. [Internet]. 2020 [citado 2021 Abr 2]; 20(4): 1241-7. Disponível em: https:// onlinelibrary.wiley.com/doi/abs/10.1111/jocd.13714.

27. Fujita J. Favorable effects of microneedling on long-standing androgenetic alopecia in an elderly man: A case report. J Cosmet Dermatol. [Internet]. 2021 [citado 2020 Abr 2]; 20(2):588-90. Disponível em: https://onlinelibrary.wiley.com/doi/abs/10.1111/ jocd.13526.

28. Sohng C, Lee EH, Woo SK, Kim JY, Park KD, Lee SJ, Lee WJ. Usefulness of home-use microneedle devices in the treatment of pattern hair loss. J Cosmet Dermatol. [Internet]. 2020 [citado 2021 Abr 2]; 20(2):591-6. Disponível em: https://onlinelibrary. wiley.com/doi/abs/10.1111/jocd.13540.
29. Gajjar PC, Mehta HH, Barvaliya M, Sonagra B. Comparative study between mesotherapy and topical $5 \%$ minoxidil by dermoscopic evaluation for androgenic alopecia in male: a randomized controlled trial. Int J Trichology. [Internet]. 2019 [citado 2021 Abr 3]; 11(2):58-67. Disponível em: https://www. ncbi.nlm.nih.gov/pmc/articles/PMC6463458/

30. Jha AK, Vinay K, Zeeshan M, Roy PK, Chaudhary RKP, Priya A. Platelet-rich plasma and microneedling improves hair growth in patients ofandrogenetic alopecia when used as an adjuvant to minoxidil. J Cosmet Dermatol. [Internet]. 2019 Jan 28. [Epub ahead of print].

31. Shah KB, Shah AN, Solanki RB, Raval RC. A comparative study of microneedling with platelet-rich plasma plus topical minoxidil (5\%) and topical minoxidil (5\%) alone in androgenetic alopecia. Int J Trichology. [Internet]. 2017 [citado 2021 Abr 3]; 9(1):14-8. Disponível em: https://www.ncbi.nlm.nih.gov/pmc/ articles/PMC5514790/.

32. Tosti A, Duque-Estrada B. Treatment strategies for alopecia. Expert Opin Pharmacother. [Internet]. 2009 [citado $2021 \mathrm{Fev}$ 12]; 10(6):1017-26. Disponível em: https://www.tandfonline. com/doi/abs/10.1517/14656560902876368.

33. Mella JM, Perret MC, Manzotti M, Catalano HN, Guyatt G. Efficacy and safety of finasteride therapy for androgenetic alopecia: a systematic review. Arch Dermatol. [Internet]. 2010 [citado 2021 Jan 27]; 146(10):1141-50. Disponível em: https://jamanetwork.com/journals/jamadermatology/articleabstract/422032.

34. Schweiger ES, Boychenko O, Bernstein RM. Update on the pathogenesis, genetics and medical treatment of patterned hair loss. J Drugs Dermatol. [Internet]. 2010 [citado 2021 Abr]; 9(11):1412-9. Disponível em: https://europepmc.org/article/ $\mathrm{med} / 21061765$.

35. Kim YS, Jeong KH, Kim JE, Woo YJ, Kim BJ, Kang H. Repeated microneedle stimulation induces enhanced hair growth in a murine model. Ann Dermatol. [Internet]. 2016 [citado 2021 Maio 20]; 28(5):586-92. Disponível em: https://www.ncbi.nlm. nih.gov/pmc/articles/PMC5064188/.

36. Jeong K, Lee YJ, Kim JE, Park YM, Kim BJ, Kang H. Repeated microneedle stimulation induce the enhanced expression of hair-growth-related genes. Int J Trichology. [Internet]. 2012 [citado 2021 Maio 20];4:117-30.

37. Wipf A, Boysen N, Hordinsky MK, Dando EE, Sadick N, Farah RS. The rise of transcutaneous drug delivery for the management of alopecia: a review of existing literature and an eye towards the future. J Cosmet Laser Ther. [Internet]. 2019 [citado 2021 Abr 2]; 21(5):247-54. Disponível em: https://www. tandfonline.com/doi/abs/10.1080/14764172.2018.1525743.

38. Almohanna HM, Perper M, Tosti A. Safety concerns when using novel medications to treat alopecia. Expert Opin Ddrug Saf. [Internet]. 2018 [citado 2021 Abr 2]; 17(11):1115-28. Disponível em: https://www.tandfonline.com/doi/abs/10.1080/14740338.2 018.1533549

39. Yu AJ, Luo YJ, Xu XG, Bao LL, Tian T, Li Z. et al. A pilot split-scalp study of combined fractional radiofrequency microneedling and $5 \%$ topical minoxidil in treating male pattern hair loss. Clin Exp Dermatol. [Internet]. 2018 [citado $2021 \mathrm{Abr}$ 2]; 43(7):775-81. Disponível em: https://onlinelibrary.wiley.com/ doi/abs/10.1111/ced.13551.

Trabalho recebido: $18 / 04 / 2021$

Trabalho aprovado: 25/10/2021

Trabalho publicado: 29/10/2021

Editor Responsável: Prof. Dr. Eitan Naaman Berezin (Editor Chefe) 